\title{
Article \\ Improving Readability of Online Privacy Policies through DOOP: A Domain Ontology for Online Privacy
}

\author{
Dhiren A. Audich, Rozita Dara *(D) and Blair Nonnecke \\ School of Computer Science, University of Guelph, 50 Stone Road, Guelph, ON N1G 2W1, Canada; \\ dhiren@audich.net (D.A.A.); nonnecke@uoguelph.ca (B.N.) \\ * Correspondence: drozita@uoguelph.ca
}

check for

updates

Citation: Audich, D.A.; Dara, R.; Nonnecke, B. Improving Readability of Online Privacy Policies through DOOP: A Domain Ontology for Online Privacy. Digital 2021, 1 , 198-215. https://doi.org/10.3390/ digital1040015

Received: 10 August 2021

Accepted: 15 November 2021

Published: 22 November 2021

Publisher's Note: MDPI stays neutral with regard to jurisdictional claims in published maps and institutional affiliations.

Copyright: (c) 2021 by the authors. Licensee MDPI, Basel, Switzerland. This article is an open access article distributed under the terms and conditions of the Creative Commons Attribution (CC BY) license (https:// creativecommons.org/licenses/by/ $4.0 /)$.

\begin{abstract}
Privacy policies play an important part in informing users about their privacy concerns by operating as memorandums of understanding (MOUs) between them and online services providers. Research suggests that these policies are infrequently read because they are often lengthy, written in jargon, and incomplete, making them difficult for most users to understand. Users are more likely to read short excerpts of privacy policies if they pertain directly to their concern. In this paper, a novel approach and a proof-of-concept tool are proposed that reduces the amount of privacy policy text a user has to read. It does so using a domain ontology and natural language processing (NLP) to identify key areas of the policies that users should read to address their concerns and take appropriate action. Using the ontology to locate key parts of privacy policies, average reading times were substantially reduced from $29-32 \mathrm{~min}$ to $45 \mathrm{~s}$.
\end{abstract}

Keywords: online privacy; privacy policy; ontology; natural language processing; human-computer interaction

\section{Introduction}

Many of the online activities place the cost of privacy on the users by requiring them to disclose their personal data in exchange for services. Due to the near permanent nature of the Internet, it results in a loss of privacy and can have a long-lasting effect on the user. A Pew Research Centre survey in 2015 found that a large percentage (91) of American adults had indicated that they feel they have lost control of the use of their private information [1]. The collection of personal data by online service providers is often justified with claims of creating a more user-centric web experience. However, personal data are sold and shared frequently with third parties that use it to profile users and track them across domains. Many surveys and studies suggested that users are increasingly concerned about their privacy online [2]. To help with users' concerns and enhance trust, companies have implemented privacy enhancing technologies (PET). These technology solutions include reducing personal information collection in terms of volume; anonymization and de-identification of personal data; opt-out mechanisms; and 'layered' policies [3,4]. Privacy policies have continued to be opaque and verbose legal documents.

Privacy policies are intended to inform users about the collection, use, and dissemination of their private data. They are also aimed to reduce the concern of users [5]. Regulations recommend that privacy policies should disclose extent and nature of information collection [6-9]. Unfortunately, most policies are often lengthy, difficult and time-consuming to read, and as a result are infrequently read [2,10-12]. The demotivating nature and the difficulty of reading privacy policies amounts to a lack of transparency. Failing to provide usable privacy policies prevents users from making informed decisions and can lead them to accept terms of use jeopardizing their privacy and personal data.

This paper proposes an approach to reduce the amount of text a user has to read using a domain ontology to identify key areas that address their concerns and allow them to take appropriate action. The approach consists of constructing a domain ontology for 
online privacy (DOOP), an ontology for online privacy policies, validated against Carnegie Mellon University's (CMU) OPP-115 data set [13] of annotated policies by domain experts. DOOP resulted in 69-99\% reductions in reading for the three sample queries that were tested. Reducing the reading time will encourage users to read privacy policies and make informed decisions online.

It is important to note that DOOP is the first ontology to capture the vocabulary of online privacy policies. It also provides a method to describe the vocabulary in terms of the privacy categories that are widely used by the Federal Trade Commission (USA) and directives proposed by other commissions in Europe and Canada.

\section{Background}

To aid users, several attempts have been made to simplify policies. Platform for Privacy Preferences (P3P) was one of the early efforts [14,15] to propose the format and use of machine-readable privacy policies. The intent was that the standardized format would make it easier to extract relevant information with the help of logic systems, e.g., reasoners. P3P had limited industry and developer participation which resulted in limited adoption of this solution. This approach also had some shortcomings including its inability to validate policies which limited developers to create effective policies [16].

Automation and crowdsourcing can reduce the cost of creating and maintaining such a data set, and still maintain reasonable quality. Terms of Service; Did not Read (ToS;DR) [17] is a project that uses crowdsourced annotations to answer key questions about the policies. The crowdsourcing approach requires large scale participation rate to ensure success of the project. This requirement can delay the effort. To address this, researchers [18,19] have made an attempt to combine machine learning and ToS;DR natural language processing (NLP) techniques to automatically evaluate the content of privacy policies and find problematic content. These methods are only effective if there is access to high quality, reliable, annotated, and up-to-date crowdsourced data which is presently lacking due to the inherent de-motivating nature of reading privacy policies [18].

In the research conducted by Ramnath et al., the researchers proposed combining machine learning and crowdsourcing (for validation) to semi-automate the extraction of key privacy practices [20]. Through their preliminary study they were able to show that non-domain experts were able to find an answer to their privacy concerns relatively quickly ( $\sim 45$ s per question) when they were only shown relevant paragraphs that were most likely to contain an answer to the question. They also found that answers to privacy concerns were usually concentrated rather than scattered all over the policy. This is an important finding because it means that if users are directed to relevant sections in the policy they should be able to address their privacy concerns relatively quickly.

In a user study conducted by Wilson et al., (2016) [13], the quality of crowdsourced answering of privacy concerns was tested against domain experts with particular emphasis on highlighted text. The researchers found that highlighting relevant text had no negative impact on accuracy of answers. They also found out that users tend not to be biased by the highlights and are still likely to read the surrounding text to gain context and answer privacy concerning questions. They also found an $80 \%$ agreement rate between the crowdsourced workers and the domain experts for the same questions [21]. These findings suggest that highlighting relevant text with appropriate keywords can provide some feedback to users inclined to read shorter policies. One way to automatically highlight relevant text in privacy policies, in a manner that can be easily scaled, is using semantic technologies (ST) such as an ontology.

There have been recent attempts to address some of the challenges related to privacy policy readability, analysis, and summarization. For instance, deep learning methods were used for automated analysis and presentation of privacy policies (e.g., Polisis tool [22]). This method was also used to build chatbots, a free form question and answering system [23]. Privacy policy evaluation and summarization methods such as natural language processing and machine learning were proposed in a few existing studies [24-26]. Despite the effort, 
the use of ontology to highlight or summarize the content of privacy policies has not been fully explored. This paper evaluates the use of ontologies to enhance the readability of these legal documents.

\section{Motivation}

As established in the introduction, online privacy policies remain unusable to average users due to their length and elusive language. This contributes to a lack of transparency which in turn leads to uninformed decisions and risk-averse behaviour. Policies that are usable tend to be read more often and give the users more confidence in sharing their personal information. This suggests that a usable privacy policy benefits all parties. Research shows that policies which highlight sections that directly address the user's concerns tend to be read more often, as it reduces the reading cost. Hence, a solution is required which considers concerns of all stakeholders, i.e., the online service providers that create privacy policies, as well as the users that do not necessarily like reading them. To avoid push-back, the solution must not require the online service providers to change their policies drastically, but must reduce the amount of text users have to read, and direct users to the text pertaining directly to their concerns.

In order to direct users to the relevant text within the policies, there needs to be a way to evaluate the text and highlight all relevant sections. Since the language being used within the policies differs so greatly $[11,19]$ among privacy policies, there needed to be a system that is able to capture all the variations of a topic. Semantic technologies such as ontologies are a well-known way of mining text and reasoning. Since domain ontologies can capture the vocabulary of a domain and specify rules about each term, it is possible to capture the diversity of concepts within the online privacy domain and reason over them to find equivalent terms for analysis. Through NLP, it is possible to logically break apart the text in a policy, and working in conjunction with the ontology, be able to recognize relevant sections within policies. This paper proposes building an ontology that is easy to build, maintain, and relatively inexpensive to scale.

\section{Ontology Engineering}

It is generally accepted that ontologies have two basic features [27]:

1. A taxonomy of terms used to name and describe the objects (concepts) being described by the ontology.

2. A specification, grounded in logic, used to add meaning between terms.

Ontologies may describe a wide variety of things in a domain, but they all share a common set of attributes [28]:

- Classes capture the core vocabulary that is used to describe a domain. They are also referred to as concepts, and are generally arranged in a hierarchical or taxonomical form as classes and sub-classes.

- Relations are definitions of how concepts inter-relate to one another.

- Attributes are the properties associated with classes that describe the features of that class.

- Formal axioms are logical statements that always evaluate to true.

- Functions are a special case of relations.

- Instances are elements of a class; and are also called individuals. Not all ontologies must have these; but if they do then that ontology constitutes a knowledge base.

There are many different types of ontologies that differ based on not only their purpose but also their content. The purpose of the ontology is determined by how widely it is meant to be used and the content is determined by the richness of the term definitions.

Since the invention of ontologies in the early 1990s, ontology engineering has remained more of an art form rather than an engineering process with rigid rules [29]. Which is to say, there is no one correct way of creating an ontology, rather the development differs depending on the ontology engineer and its purpose. However, several methodologies 
have been proposed to standardize the process of creating ontologies. Among those, Ontology 101 [30] and NeOn [31] are two of the commonly used ontology engineering methods. Ontology 101 provides a step-by-step methodology for creating simple ontologies iteratively using the Protégé-2000 [32] ontology engineering tool, developed by Mark Musen's research group at Stanford Medical Informatics. Since this methodology is geared towards the Protégé tool, it focuses more on declarative frame-based systems used to describe objects in a domain along with their properties rather than more complex and domain specific ontologies that can be constructed with the other methodologies. The NeOn methodology promotes reusing and combining ontologies to create new and networked ontologies drawing on multiple ontologies for their knowledge. NeOn provides a scenariobased framework to create ontologies and develop and expand ontology networks. Rather than a rigid work flow like Ontology 101, NeOn prescribes a set of guidelines for multiple alternative processes for various stages of ontology development that may change with the design decisions.

There are a few ways of evaluating an ontology, each depending on the type and purpose of the ontology constructed. One of the practical ways of evaluating ontologies is a data driven approach. In this approach, ontologies are simply compared with the sources of data from the domain that the ontology is meant to cover. This involves statistically extracting key terms and concepts from the corpus the ontology is meant to cover and evaluating if they exist in the ontology itself. This is done via calculation of the precision and recall scores [33-36].

Precision $(P)$ is the ratio of the number of relevant terms returned from a term extraction algorithm (\{manually selected $\} \cap\{$ machine-selected $\}$ ) to the total numbers of retrieved terms by the algorithm (\{machine-selected\}). The precision is calculated using Equation (1).

$$
\mathrm{P}=\frac{\mid\{\text { manually selected }\} \cap\{\text { machine-selected }\} \mid}{\mid\{\text { machine-selected }\} \mid}
$$

The recall $(R)$ of an information system is defined as the ratio of the number of relevant terms returned to the total number of relevant terms in the collection. The recall is computed using Equation (2).

$$
\mathrm{R}=\frac{\mid\{\text { manually selected }\} \cap\{\text { machine-selected }\} \mid}{\mid\{\text { manually selected }\} \mid}
$$

Formal competency questions (CQs), an evaluation strategy proposed by $[37,38]$, is another ontology validation method. In this method, informal competency questions (queries) are first expressed formally. These questions are requirements that are in the form of questions that an ontology must be able to answer. The formal questions are then evaluated using completeness theorems with respect to first (axioms) and second order (situational calculus) logic representation of concepts, attributes, and relations.

\section{Methodology}

The end goal of DOOP is the creation of a tool, e.g., a browser extension, that uses the ontology as a knowledge base to parse online privacy policies and highlight sections in the policy that would addresses a user's privacy-related queries. To that end, a hybrid construction approach was used, a combination of Ontology 101 and NeOn methodologies in conjunction with iterative development. Ontology 101 was proposed with the intent of using Protégé for the construction of ontology. Since the latest version of Protégé [39] was used in the construction of DOOP, Ontology 101 was used as the prime methodology. Furthermore, ontology engineering guidelines provided under NeOn's Scenario 1 (From Specification to Implementation), which includes steps from other methodologies, were used as the foundation to specify and build DOOP. Instead of building a complete ontology that exhaustively considers every possible case, DOOP was iteratively built in iterations as described by RapidOWL. By expanding the vocabulary one query at a time the ontology 
remains open and malleable enough such that future developments require relatively less effort to alter the structure of the ontology as needed. The ontology was implemented in OWL-DL using the Protégé tool (version 5.2.0) for ontology engineering.

The language between privacy policies is inconsistent. These inconsistencies meant that the corpus of privacy policies used for ontology development had to be large and diverse to capture as many terms as possible, and from different economic zones. For this reason, we took a two-step approach to extract keywords and validate the ontology. First, the key terms extracted from a corpus of 631 privacy policies were gathered [40]. Seven classes were identified: data collection, data retention, data security, data sharing, target audience, user access, and user choice. These were identified in the work done by [41]; and were based on the logical division of policies described under the FIPPs (Fair Information Practice Principles), and principles identified under OECD's guidelines for (Organization for Economic Co-operation and Development) protection of privacy and data flows [42]. These classes are commonly found in both cookie and privacy policies. The hierarchy of the ontology was developed as needed to satisfy the CQs. Subsequently, CMU's OPP-115 corpus, a corpus of 115 manually annotated privacy policies with 23,000 data practices was used to validate DOOP. Lastly, the goal of building DOOP was to support the following end-users: privacy researchers, NLP experts who are interested in doing work in the online privacy domain, and software developers who would like to use an ontology to create tools for the online privacy domain. An overview of DOOP construction and validation is shown in Figure 1.

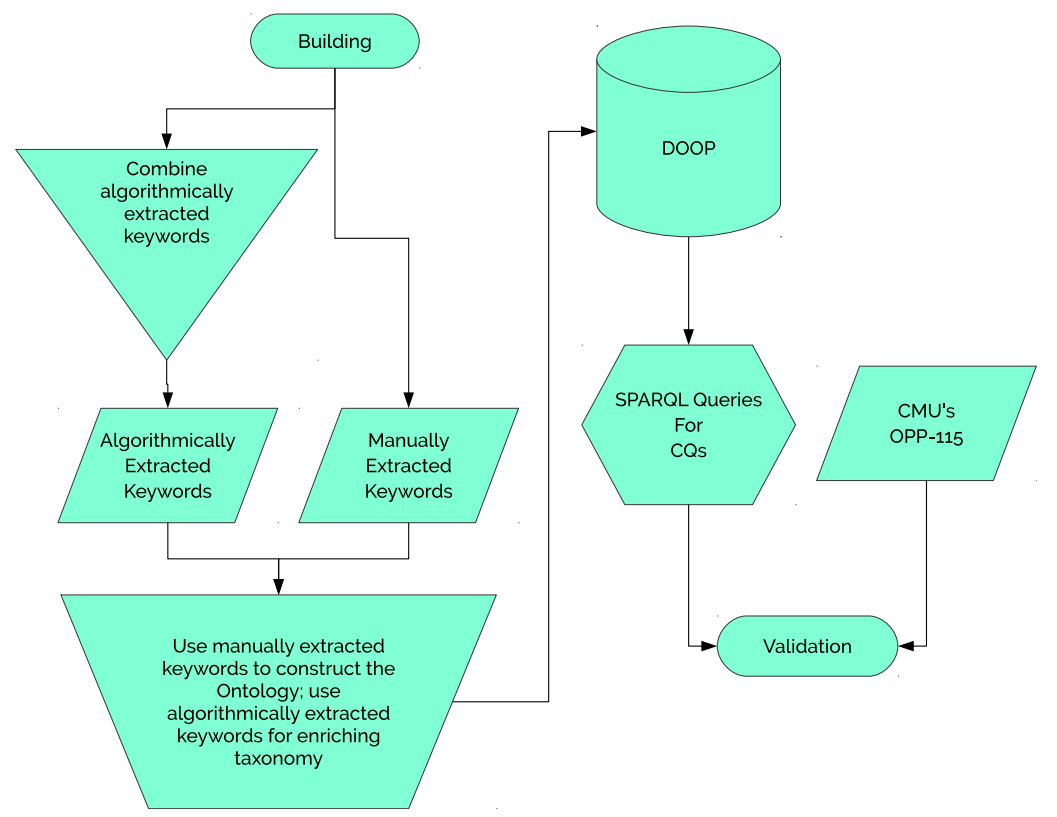

Figure 1. Overview of how DOOP was constructed and validated.

\section{Competency Questions}

Competency questions are a set of queries that the ontology should be able to answer based on its axioms. This is why they are used for not only defining ontology requirements but also ontology evaluation; the result from a CQ can be used to determine the correctness of an ontology. CQs can be used for evaluation either manually or automatically through the use of SPARQL queries. DOOP was constructed and evaluated through the use of CQs. After defining a CQ, the ontology was constructed iteratively by defining as many axioms needed to answer the CQ. The following 3 CQs were used for constructing the ontology:

1. Does this website share my personal information with third-parties?

2. Does this website use tracking cookies?

3. Can I opt-in/opt-out of information gathering? 
The first query was selected based on the most common concern users report, a worry / concern about their personal information being shared with unauthorized and unintended parties. It is also a question that every privacy policy is designed to address. The other two queries are more specific and hence were chosen to capture narrower results.

\section{Results}

A breakdown of DOOP is given in Table 1. An example of a class is given in Table 2.

Table 1. Breakdown of DOOP.

\begin{tabular}{cc}
\hline Property & Count \\
\hline Axioms & 304 \\
Logical axiom count & 171 \\
Declaration axioms count & 71 \\
Class count & 15 \\
Object property count & 6 \\
Data property count & 10 \\
Individual count & 35 \\
Annotation property count & 8 \\
\hline
\end{tabular}

Table 2. Example of a class in DOOP.

\begin{tabular}{cc}
\hline Preferred Name & Personally Identifiable Information \\
Alternative & PII \\
Description & Information about an individual person, organization, or any other entity. \\
SubClass of & Keyword \\
Instances & Email Address, Email Preference, Name, Age, PII \\
\hline
\end{tabular}

\subsection{Top Structure}

The ontology is divided into four main classes derived from the standard OWL root class for everything owl:Thing: Keyword, PrivacyCategory, PrivacyPolicy, and Question. The following are rules for creating the rest of the classes and individuals. Refer to Figures 2 and 3.

1. The Keyword class captures most of the vocabulary contained in DOOP. As shown in Figure 2, it is a sub-class of owl:Thing. Classes act as sets of individuals, hence, Opt-in and Opt-out form instances of Keyword (Figure 3) but Cookie is a sub-class of Keyword; this is because the class Cookie has the instances, 'Do Not Track' and 'Web Beacon', which are types of cookies. Legal Act, Country, and Organization, are all subclasses of Keyword which are interrelated by relationships: 'Applies To', 'Operates In', 'Enacted', and 'Based'. Legal acts 'Applies To' country which is an inverse of 'Enacted'. Similarly, organizations 'Operates In' countries, and countries serve as 'Base' for organizations which is also an inverse relationship to the former relationship.

2. Privacy category class does not have a sub-class, but has 7 individuals: data collection, data retention, data security, data sharing, target audience, and user access. Both Keyword and Question classes share a 'Related To' relationship with Privacy Category, since keywords and questions are logically classified under various privacy categories. An example of this is shown in Figure 3, where Opt-Out is 'Related To' User Choice, Data Collection, and Data Sharing. Since, Opt-Out is 'Similar To' Opt-In, it is inferred that it too is related to the three categories.

3. The Question class is where all of the questions are stored as individuals. This class has no sub-class because all of the questions are types of Question. Additional information is stored as object properties, e.g., concern, competency question, and SPARQL query.

4. The Privacy Policy class stores metadata for privacy policies already processed. It is divided into two sub-classes: Policy Document and Cookie Policy. Privacy policies are individuals of the Policy Document class, and if a separate coolie policy exists then its metadata are stored under the Cookie Policy class. 


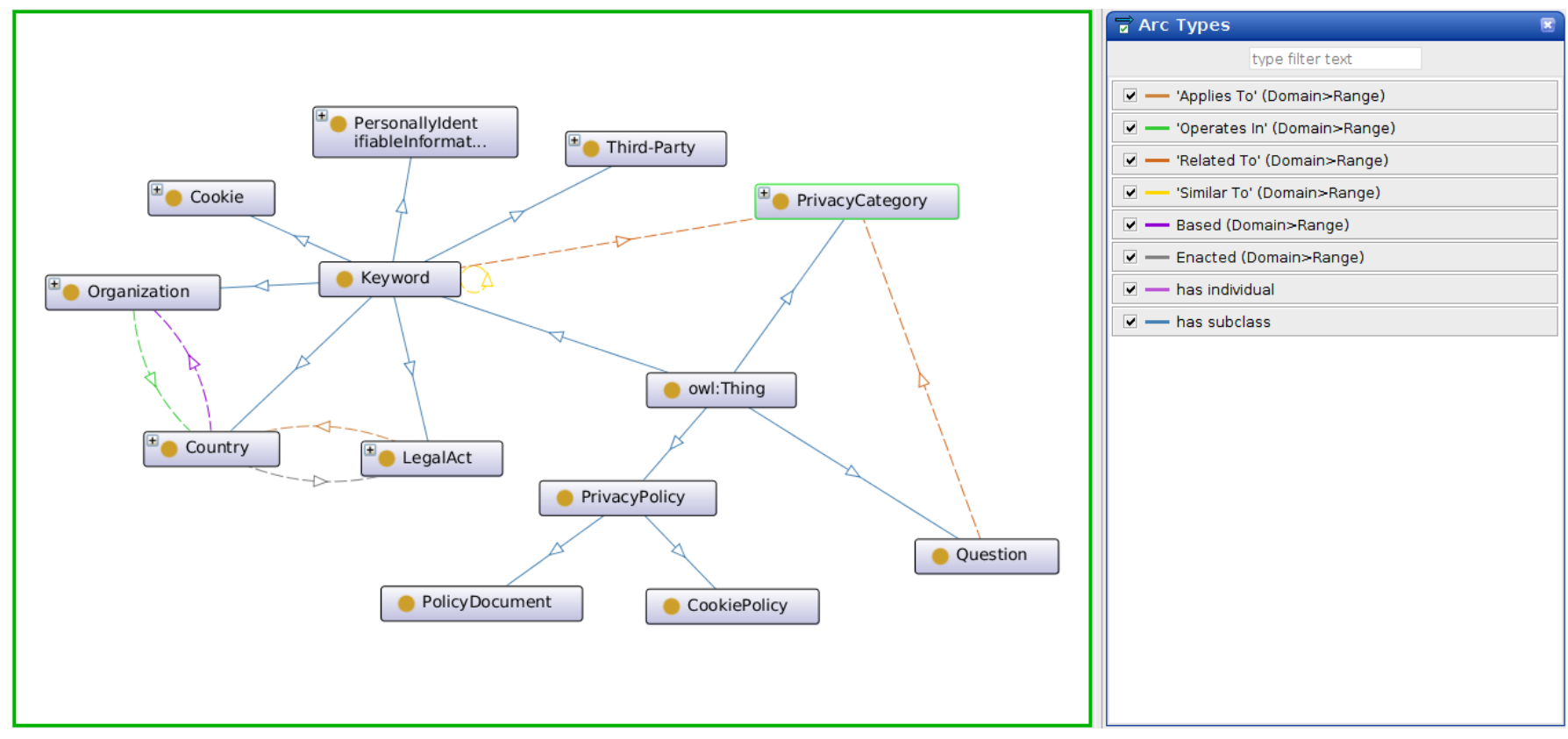

Figure 2. Top class structure of DOOP. Some classes and individuals are not shown due to space limitations.

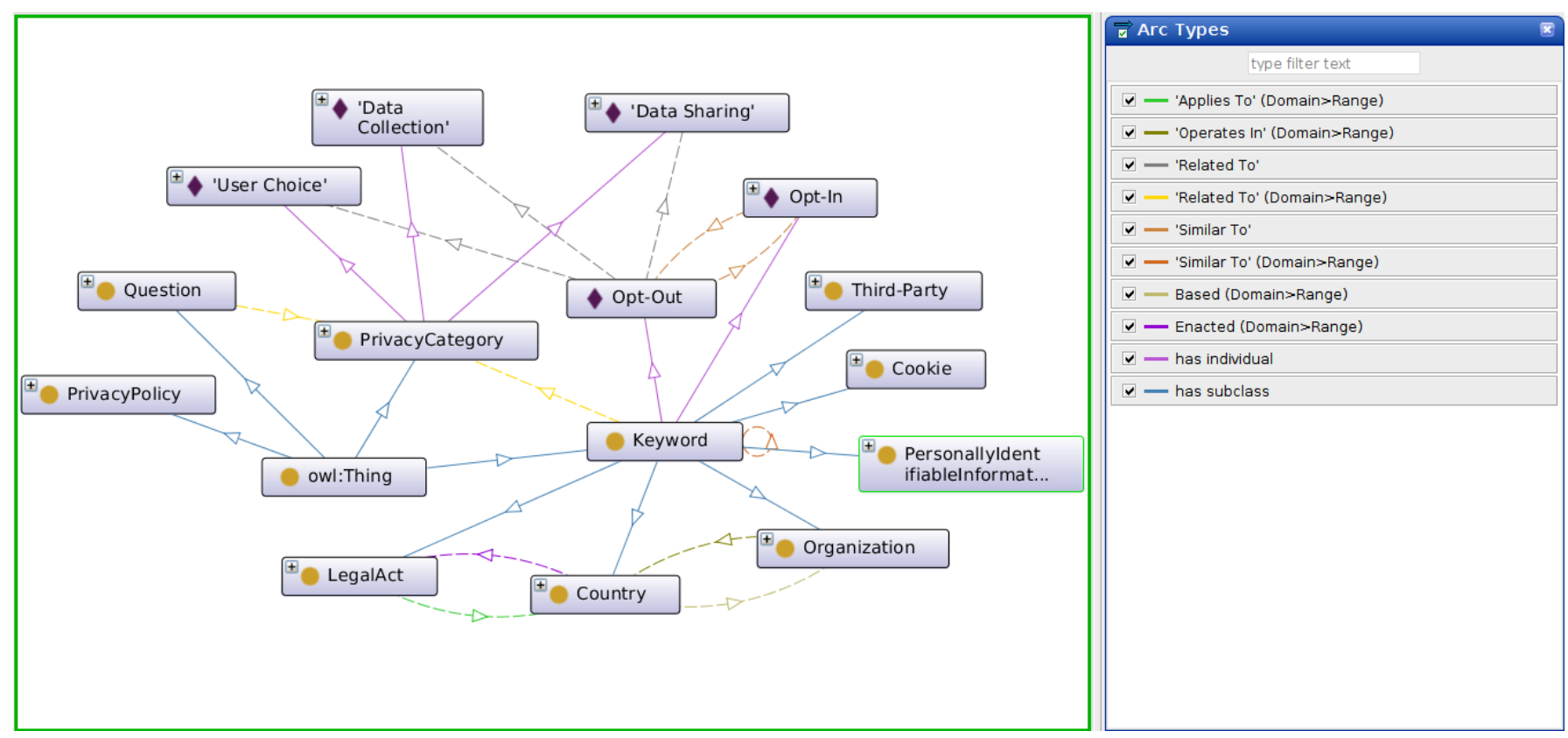

Figure 3. Example of individuals in DOOP. Some classes and individuals are not shown due to space limitations.

The general structure of the ontology was developed with the firm intention of developing a tool to dissect an online privacy policy into sections that the user might be most interested in based on their concerns and the captured vocabulary offered by the domain ontology. Since, to the best of our knowledge, no previous attempt had been made to capture only the vocabulary in online privacy policies, there were no ontologies to refer to for creating classes and structure.

\subsection{Inference and Structure}

DOOP is primarily composed of a single is- $a$ asserted inheritance structure, expressed with subclass relations in OWL-DL. However, other relations also exist to enable further development and to capture more complex logic. An exhaustive list of object relations along with their properties is described in Table 3. These provide useful classification hierarchies and extendability for the users of the ontology. These relationships allow the user to infer 
which keywords are related to what privacy category and question; which questions share a certain number of keywords, useful for recommendation; what legal acts are enforceable in a country; what organizations enforce which legal acts and where they are located; determine the overlap of vocabulary between privacy categories. DOOP is consistent with all three reasoners in Protégé 5.2.0: FaCT++ [43], HermiT [44], and Pellet [45].

Table 3. Object properties presently available in DOOP.

\begin{tabular}{cccc}
\hline Relationship & Domain & Range & Characteristics \\
\hline is a & $\infty$ & $\infty$ & Transitive \\
superclass of & $\infty$ & $\infty$ & Functional, \\
applies to & LegalAct & Country & InverseOf:enacted \\
based & Country & Organization & InverseOf:OperatesIn \\
enacted & Country & LegalAct & InverseOf:AppliesTo \\
operates in & Organization & Country & Functional, \\
related to & Keyword, Question & PrivacyCategory & InverseOf:Based \\
similar to & Keyword & Keyword & Transitive, Symmetric \\
\hline
\end{tabular}

\subsection{Validation}

Owing to the individual and subjective nature of ontologies, ontology validation is a difficult process. DOOP was validated in two ways: CQs and data driven. CMU's OPP-115 data set was used as the primary benchmark for validation. Since there were 10 policy annotators, there were many overlaps in the labelling of policies, as well as multiple labels for the same policy. To reduce redundancy, the authors of OPP- 115 consolidated annotations with three convergence thresholds: $0.5,0.75$, and 1 . These were calculated as normalized aggregated overlap of spans of text assigned the same data attribute for the same data practice identified by multiple annotators. For our validation, annotations from all three thresholds were considered. Furthermore, OPP-115 annotators have classified their annotation under 10 categories which had to be mapped to our 7 categories; Figure 4 shows this mapping. The mapping was necessary because at the time of undertaking this research OPP-115 data set had not yet been released so the categories introduced by [41] were used. For validation, four experiments were conducted that evaluated various aspects of the ontology:

1. Correctness: Compute the number of matched privacy categories for the same sentences from both DOOP (based on the keywords the sentence contains) and OPP-115.

2. Policy coverage: Compute the number of sentences that the reader has to theoretically read to understand the risks associated with their concerns.

3. Completeness: Compute per policy keywords that existed in the ontology but not in the policy.

4. Correctness: Compute cases where the keyword's assigned category in the ontology did not match the OPP-115's annotation's assigned category. 
DOOP

OPP-115

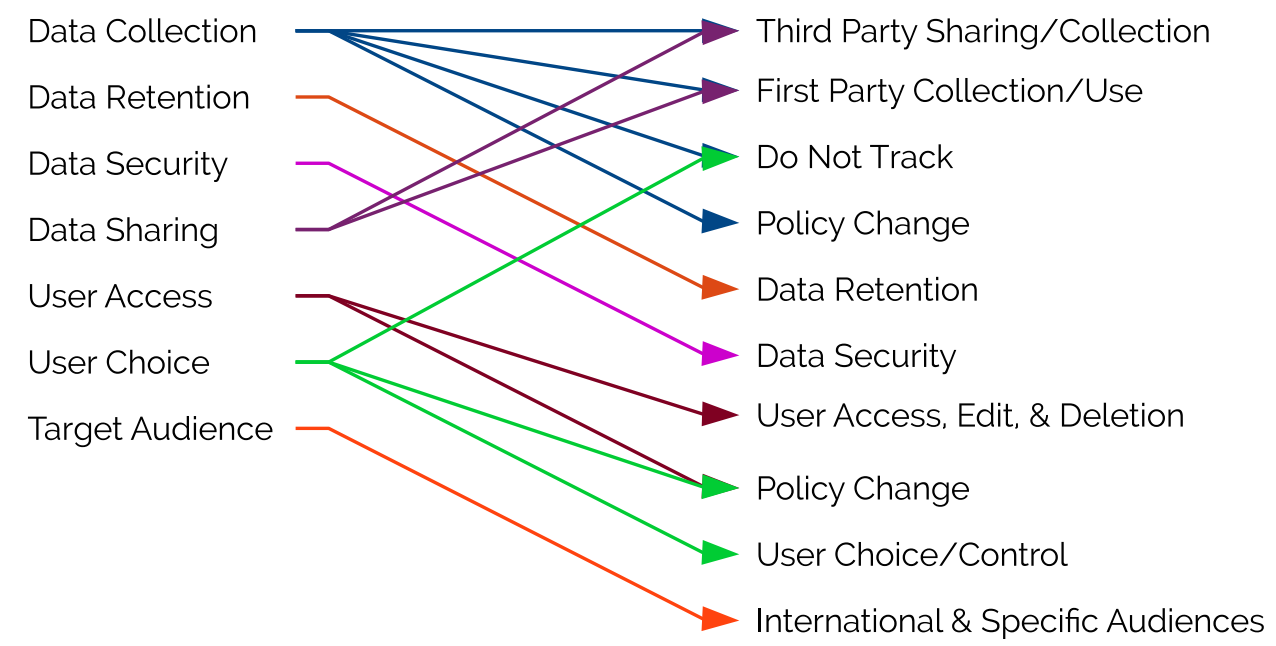

Figure 4. Mapping DOOP categories to OPP-115.

\subsubsection{Experiment 1: Correctness}

In order to compare categories, the annotation set's results had to be processed. The results are presented as a CSV file for each privacy policy in the consolidation directory as indicated by their manual. The column description used by the CSV files is presented below:

(A) annotation ID (a globally unique identifier for a data practice)

(B) batch ID (name of a batch in the annotation tool; often indicates who the annotators were)

(C) annotator ID

(D) policy ID (this corresponds to the numeric prefixes in the policy filename, as found in other directories)

(E) segment ID (the zero-indexed, sequential identifier of the policy segment; e.g., the first segment in a policy's text is segment zero)

(F) category name

(G) attribute-value pairs (represented as JSON, this where the annotations are stored)

(H) policy URL

(I) date

A qualified positive match of categories occurs when the selectedText for an annotation under attribute-value contains any of the keywords returned by DOOP for a query whose DOOP categories also match their mapped OPP-115 category name. Results for all three queries, and for all three thresholds are presented in Table 4. A sentence in a policy that contains any of the keywords that DOOP returns is denoted by SM or Sentences Matched, and sentences for which there is category match is denoted by PM or Positive Matched, while the score is the percentage.

Table 4. Results for DOOP validation, experiment 1 .

\begin{tabular}{ccccccccccc}
\hline \multirow{2}{*}{ Query } & \multicolumn{3}{c}{$\mathbf{0 . 5}$} & \multicolumn{9}{c}{$\mathbf{0 . 7 5}$} & \multicolumn{3}{c}{$\mathbf{1}$} \\
\cline { 2 - 10 } & $\mathbf{S M}$ & $\mathbf{P M}$ & $\mathbf{\%}$ & $\mathbf{S M}$ & $\mathbf{P M}$ & $\mathbf{\%}$ & $\mathbf{S M}$ & $\mathbf{P M}$ & $\mathbf{\%}$ \\
\hline 1 & 5254 & 3262 & $62.09 \%$ & 5926 & 3689 & $62.25 \%$ & 6540 & 3931 & $60.11 \%$ \\
2 & 322 & 289 & $89.75 \%$ & 368 & 331 & $89.95 \%$ & 401 & 362 & $90.27 \%$ \\
3 & 803 & 622 & $77.46 \%$ & 907 & 713 & $78.61 \%$ & 1012 & 759 & $75.00 \%$ \\
\hline
\end{tabular}




\subsubsection{Experiment 2: Coverage}

For this experiment, the average number of sentences that exist in a policy was first calculated, then it was divided by the number of sentences that contained the keywords that were returned after the execution of the SPARQL query for a privacy concern. The results for all queries as well as for all convergence thresholds are presented in Table 5. The average number of total sentences in privacy policies is denoted by TS, whereas selected sentences that contain a keyword also in the returned query from DOOP is denoted by SS.

Table 5. Results for DOOP validation, experiment 2.

\begin{tabular}{cccccccccc}
\hline \multirow{2}{*}{ Query } & \multicolumn{3}{c}{$\mathbf{0 . 5}$} & \multicolumn{3}{c}{$\mathbf{0 . 7 5}$} & \multicolumn{3}{c}{$\mathbf{1}$} \\
\cline { 2 - 9 } & TS & SS & Coverage & TS & SS & Coverage & TS & SS & Coverage \\
\hline 1 & 91.7 & 27.99 & $30.99 \%$ & 91.7 & 27.99 & $30.99 \%$ & 91.7 & 27.99 & $30.99 \%$ \\
2 & 91.7 & 1.18 & $1.42 \%$ & 91.7 & 1.18 & $1.42 \%$ & 91.7 & 1.18 & $1.42 \%$ \\
3 & 91.7 & 4.12 & $4.32 \%$ & 91.7 & 4.12 & $4.32 \%$ & 91.7 & 4.12 & $4.32 \%$ \\
\hline
\end{tabular}

\subsubsection{Experiment 3: Completeness}

In this experiment, the number of keywords that the ontology returned for a particular query that did not exist in the privacy policies was calculated. The primary purpose here was to investigate how many unique terms existed in DOOP that did not exist in the policy. Since all of 115 policies used in OPP-115 were American, unique terms found in DOOP would indicate a geographic non-specific ontology that can be generally used in most English speaking countries. Results from this experiment are reported in Table 6. The average number of keywords found is denoted by KF, and average number of keywords not found is denoted by NF.

Table 6. Results for DOOP validation, experiment 3.

\begin{tabular}{cccccccccc}
\hline \multirow{2}{*}{ Query } & \multicolumn{3}{c}{$\mathbf{0 . 5}$} & \multicolumn{3}{c}{$\mathbf{0 . 7 5}$} & \multicolumn{3}{c}{$\mathbf{1}$} \\
\cline { 2 - 10 } & KF & NF & Unique & KF & NF & Unique & KF & NF & Unique \\
\hline 1 & 3.86 & 9.14 & $70.31 \%$ & 3.86 & 9.14 & $70.31 \%$ & 3.86 & 9.14 & $70.31 \%$ \\
2 & 0.41 & 2.59 & $86.33 \%$ & 0.41 & 2.59 & $86.33 \%$ & 0.41 & 2.59 & $86.33 \%$ \\
3 & 1.39 & 1.61 & $53.67 \%$ & 1.39 & 1.61 & $53.67 \%$ & 1.39 & 1.61 & $53.67 \%$ \\
\hline
\end{tabular}

\subsubsection{Experiment 4: Correctness}

Since, assigning categories to keywords is a manual task, and privacy categories from DOOP were further mapped onto category names assigned by annotators from OPP115, we wanted to know how often we differed in opinion. Thus, in this experiment we investigated how often keyword assigned category in DOOP differs from OPP-115. Results from this experiment is presented in Table 7.

Table 7. Results for DOOP validation, experiment 4 .

\begin{tabular}{cccccccccc}
\hline \multirow{2}{*}{ Query } & \multicolumn{3}{c}{$\mathbf{0 . 5}$} & \multicolumn{9}{c}{$\mathbf{0 . 7 5}$} & & \multicolumn{3}{c}{$\mathbf{1}$} \\
\cline { 2 - 10 } & Total & Diff & $\%$ & Total & Diff & \% & Total & Diff & \% \\
\hline 1 & 5254 & 1992 & $37.91 \%$ & 5926 & 2237 & $37.75 \%$ & 6540 & 2609 & $39.89 \%$ \\
2 & 360 & 78 & $21.67 \%$ & 415 & 91 & $21.93 \%$ & 465 & 110 & $23.66 \%$ \\
3 & 1606 & 984 & $61.27 \%$ & 1814 & 1101 & $60.69 \%$ & 2024 & 1265 & $62.50 \%$ \\
\hline
\end{tabular}




\section{Discussion}

In Experiment 1 (Table 4), a mean of $76.16 \%$ match for privacy categories with a standard deviation of 12.41 was achieved. Since the OPP-115 data set was manually curated by domain experts, a high degree of match indicates a high degree of accuracy achieved by the ontology for identifying sentences in context based on the vocabulary. Now, the users need not read the entire policy, but can be directed to appropriate sentences in the policy that deal directly with their concerns with a reasonable amount of accuracy. Additionally, there is a negligible increase in the accuracy of the automatic categorization when the convergence threshold is 0.75 . This could be as consolidation reduces redundancy, without overdoing it at 1.0 convergence threshold.

One of the prime reasons that users do not read privacy policies and are left uninformed, is that they tend to be overly long. Any tool trying to fix this issue must not only find correct information but also require less reading. In Experiment 1, algorithmic assignment of privacy categories to sentences performed favorably against the manual annotations performed by domain experts. Thus, in Experiment 2, policy coverage was investigated to identify how much of a policy is the user asked to read for the three identified concerns. This experiment demonstrated (Table 5) that the user has to read on average 11.09 sentences with a standard deviation of 14.70 , or about $12.09 \%$ of a policy with a standard deviation of 16.06 to know if all of their concerns are met. Assuming that a paragraph is roughly 10 sentences, then based on research done by [20], we know that it would take roughly $45 \mathrm{~s}$ to read it. This reduced time makes the privacy policies more inviting and should encourage more users to read policies even if partially.

To further investigate these results, additional analysis of the individual results from experiments for all of the thresholds and queries was conducted. Figures 5-7 show the results of the queries for the 0.75 threshold. A radical relationship between the number of sentences selected and the length of the policies was expected, where the reading proportionally increases as the length of the policies increase, but then stabilize at some horizontal asymptote. However, this did not occur. A strong positive linear relationship was observed for the first experiment (Figure 5), no correlation for the second experiment (Figure 6), and weak positive for the third experiment (Figure 7). A qualitative analysis provided several clues for these behaviours:

1. The vocabulary in the first query, was trying to capture more than one concern. Since the ontology only returned a vocabulary of terms, it was hard to determine the correct context sometimes as one set of keywords could be used in multiple instances under different contexts. One possible solution to this problem is having the ontology also capture POS tags that determine the structure of the sentences and identifies the associated verb (e.g., sharing) and thus provide a context under which the sentence occurs. This would help distinguish one context from another where the most of the vocabulary is shared. This idea is explored in Section 7.2.

2. Policies were repeated throughout the document. This accounted for the linear relationship for the first experiment. Redundancies in the policies drove up the number of sentences to read.

3. Keywords returned by the ontology were narrowly defined. This was an important distinction with the second query regarding tracking cookies. The keyword 'cookie' was not being used because not all cookies are tracking, this meant that several cases where that term was being used to establish context were not captured. For example, "We do not use tracking cookies". would be selected, but, 'Cookies may be used to track you', would be ignored. Similar to the first problem, POS tags for some terms could be captured by the ontology to identify context as a remedy to this problem. In the OPP- 115 data set, only $30 \%$ of the policies had a 'tracking cookie' policy that was part of the privacy policy that was extracted.

4. Some policies were missing entirely. Sometimes, a supplementary document was used to state policies, e.g., 'Cookie Policy'. This supplementary document was not stored on the same page as the privacy policy; hence, it was not picked up by the 
scraper scripts. This is a difficult challenge to solve as there is no consensus as to what the URL must be for the cookie policy. However, a reasonable attempt can be made to collect this page as well and amend it to the privacy policy.

\section{Query 1: OPP-115 Threshold 0.75}

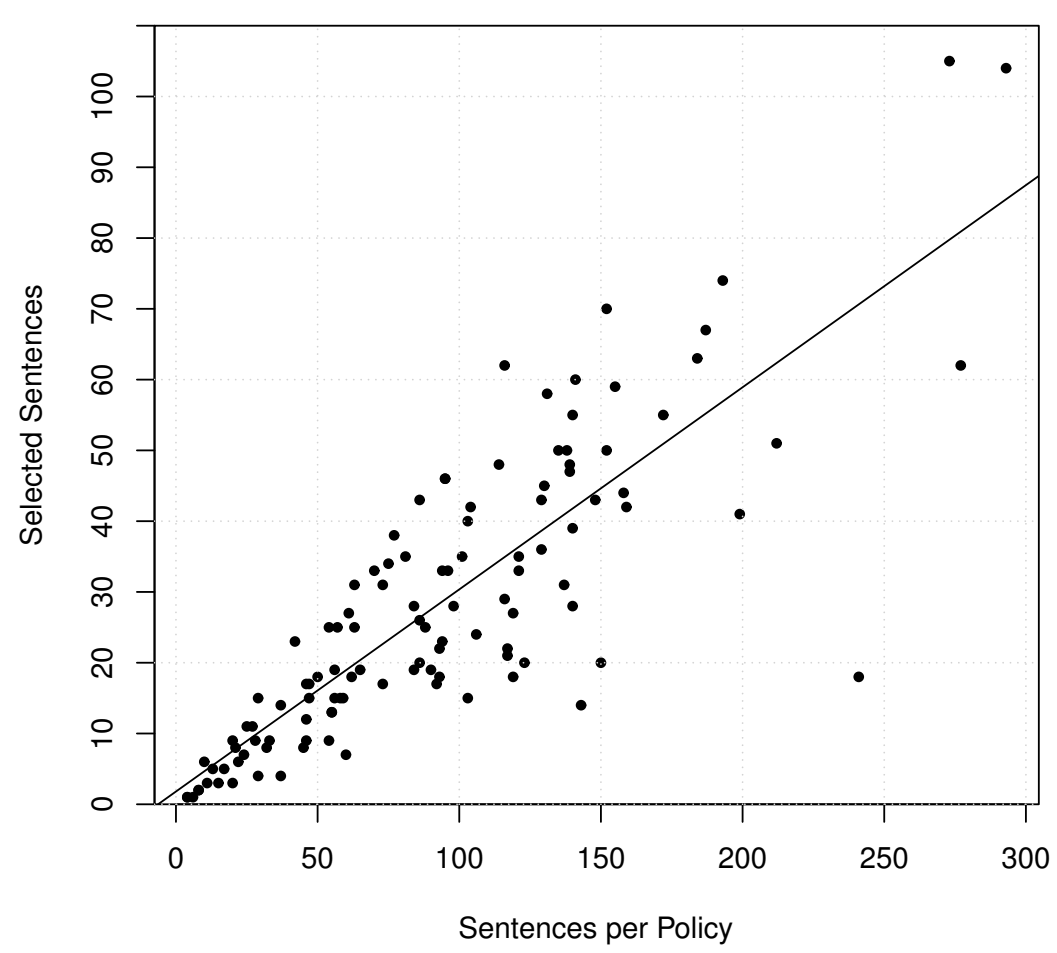

Figure 5. Sentences selected for reading for query 1: 'Does this website share my personal information with third-parties?'.

Query 2: OPP-115 Threshold 0.75

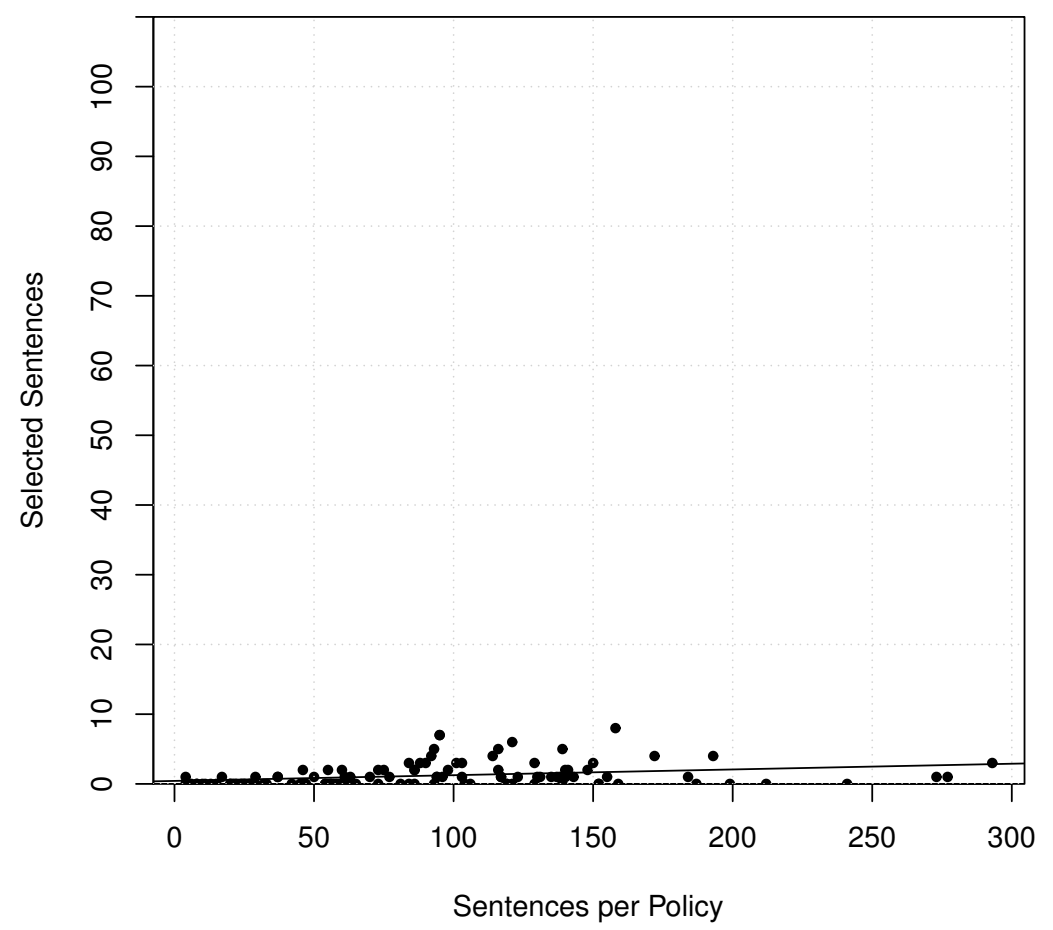

Figure 6. Sentences selected for reading for query 2: 'Does this website use tracking cookies?'. 
Query 3: OPP-115 Threshold 0.75

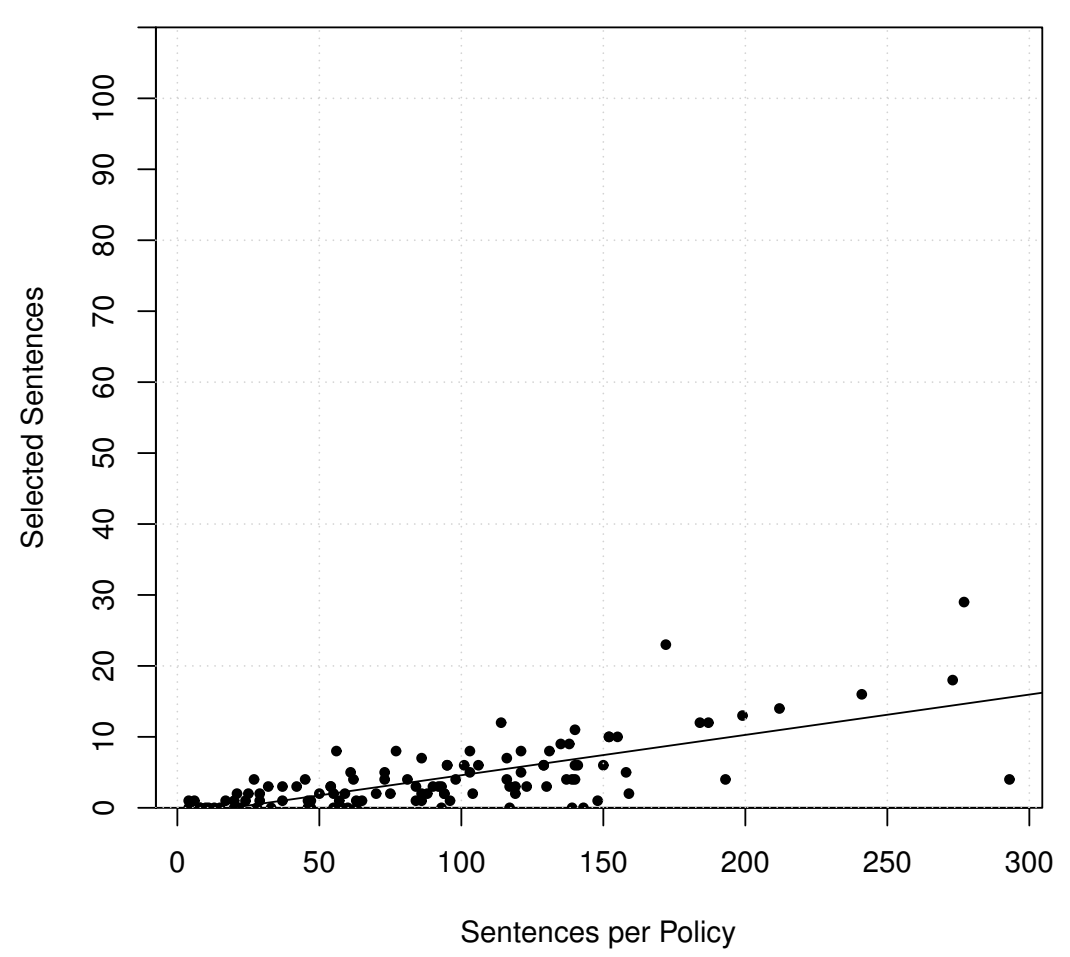

Figure 7. Sentences selected for reading for query 3: 'Can I opt-in/opt-out of information gathering?'.

In the creation of the taxonomy for DOOP, the vocabulary was not restricted to a particular geographically intended audience (in order to make the ontology as general purpose as possible). OPP-115's dataset contained only American privacy policies. Hence, there were terms in DOOP that did not exist in OPP-115. Experiment 3 was conducted to investigate the uniqueness of DOOP in comparison to OPP-115. Table 6 shows that on average $70.10 \%$ of terms are unique to DOOP with a standard deviation of 14.14 . This was expected as not just American policies were considered when extracting keywords from privacy policies, but also Canadian and European ones. The 70\% terms also include localization of the American spelling along with synonyms, hypernyms, and E.U. and Canada specific terms, which made the ontology more unique here, e.g., advertiser/advertizer, and name/full name.

Finally, Experiment 4 investigated how much the labels of keywords agreed between DOOP and OPP-115. The mean disagreement between the data sets was $40.81 \%$ with a standard deviation of 17.03. The most disagreement being with query 3 . One of the reasons for this discrepancy could be due to the mapping of categories from OPP-115 to DOOP. Since the mapping of the privacy categories between the data sets was not one-to-one, approximations had to be made. This meant that one category in one data set was mapped onto multiple categories in the other introducing a large amount of variance in the topics captured by each category. Another explanation has to do with the limited vocabulary DOOP currently captures. In its present state it was created to be a proof-of-concept system. As the vocabulary increases, it is expected that the results for all four experiments will also improve.

\subsection{Generalizing Keyword Searches}

An investigation was conducted to see what happens when more generic keywords are added to queries for focused and narrowly defined queries, such as query 2 . The term 'cookie' was added to the list of keywords for the second query, and all of the experiments re-run. The results are shown in Tables $8-11$ and Figure 8. 
Table 8. Results for DOOP validation for query 2, experiment 1.

\begin{tabular}{cccccccccc}
\hline \multirow{2}{*}{ 'Cookie' } & \multicolumn{3}{c}{$\mathbf{0 . 5}$} & \multicolumn{3}{c}{$\mathbf{0 . 7 5}$} & \multicolumn{3}{c}{$\mathbf{1}$} \\
\cline { 2 - 11 } & $\mathbf{S M}$ & $\mathbf{P M}$ & $\mathbf{\%}$ & $\mathbf{S M}$ & $\mathbf{P M}$ & $\mathbf{\%}$ & $\mathbf{S M}$ & $\mathbf{P M}$ & \% \\
\hline No & 322 & 289 & $89.75 \%$ & 368 & 331 & $89.95 \%$ & 401 & 362 & $90.27 \%$ \\
Yes & 1367 & 1157 & $84.64 \%$ & 1516 & 1295 & $85.42 \%$ & 1625 & 1380 & $84.92 \%$ \\
\hline
\end{tabular}

Table 9. Results for DOOP validation query 2, experiment 2.

\begin{tabular}{cccccccccc}
\hline \multirow{2}{*}{ Cookie' $^{$\cline { 2 - 9 }$}$} & \multicolumn{3}{c}{$\mathbf{0 . 5}$} & \multicolumn{3}{c}{$\mathbf{0 . 7 5}$} & \multicolumn{3}{c}{$\mathbf{1}$} \\
\cline { 2 - 10 } & TS & Coverage & TS & SS & Coverage & TS & SS & Coverage \\
\hline No & 91.7 & 1.18 & $1.42 \%$ & 91.7 & 1.18 & $1.42 \%$ & 91.7 & 1.18 & $1.42 \%$ \\
Yes & 91.7 & 8.12 & $9.69 \%$ & 91.7 & 8.12 & $9.69 \%$ & 91.7 & 8.12 & $9.69 \%$ \\
\hline
\end{tabular}

Q2 with 'cookie': OPP-115 Threshold 0.75

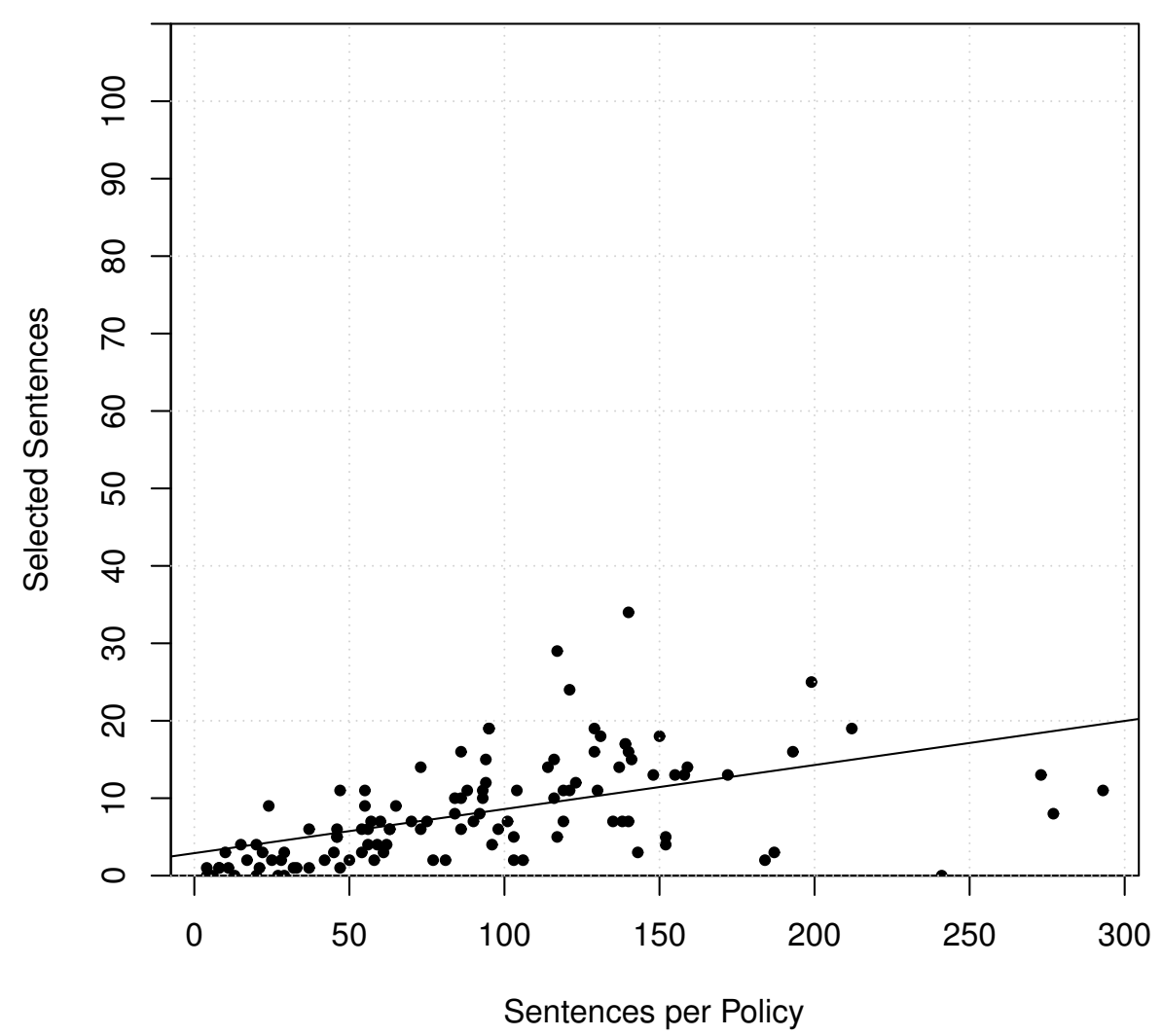

Figure 8. Results for experiment 2 for query 2 after adding 'cookie' to the keywords.

Table 10. Results for DOOP validation for query 2, experiment 3.

\begin{tabular}{cccccccccc}
\hline \multirow{2}{*}{ ‘ookie' } & \multicolumn{3}{c}{$\mathbf{0 . 5}$} & \multicolumn{4}{c}{$\mathbf{0 . 7 5}$} & \multicolumn{3}{c}{$\mathbf{1}$} \\
\cline { 2 - 10 } & KF & NF & Unique & KF & NF & Unique & KF & NF & Unique \\
\hline No & 0.41 & 2.59 & $86.33 \%$ & 0.41 & 2.59 & $86.33 \%$ & 0.41 & 2.59 & $86.33 \%$ \\
Yes & 1.04 & 2.96 & $74.00 \%$ & 1.04 & 2.96 & $74.00 \%$ & 1.04 & 2.96 & $74.00 \%$ \\
\hline
\end{tabular}




\subsection{Contextualising Keyword Searches}

In general, the total number of sentences dramatically increased from 364 to 1503 (Table 8), and the accuracy went from $90 \%$ to $85 \%$. This was expected as 'cookies' was mentioned more often because they are used for more than just tracking. They are also widely used for the storage of temporary data. This can be also observed in Table 10, which shows there is at least one word common to the vocabulary in the ontology and is consistently being found in the policies. Furthermore, this resulted in an increase in the estimated number of sentences to read per policy (on average going from 1.18 to 8.12 Table 9). The addition of non-specific keywords also increased the variability of the sentences to read, as can be observed in Figure 8. This also led to fewer policies being flagged as having 0 sentences to read. Once again, the number of sentences in a policy and the amount of reading a user has to do is linearly correlated. One of the most important measure is the increased disagreement between the recommended and the annotated sentences (Table 11). This indicates that adding generic terms deteriorates the overall quality of the recommendations. The indicators in this short study demonstrates that in order for the recommended reading to be useful to the user, it must have fewer generic terms and more targeted ones.

After a qualitative review of the sentences that were selected for Query 1 in Experiment 2, it was found that sentences were broadly selected to capture any mention of attributes associated with personal information and not necessarily-third-party sharing. To reduce the number of sentences selected for reading in Experiment 1, a simple experiment was conducted where contextual keywords were used to further limit the types of sentences that were only associated with 'third-party', 'disclose' and 'sharing'. Only sentences with at least one of those keywords mentioned were chosen. The results of this The experiments are reported in Table 12 and Figure 9.

Query 1 with Contextual Keywords: OPP-115 Threshold 0.75

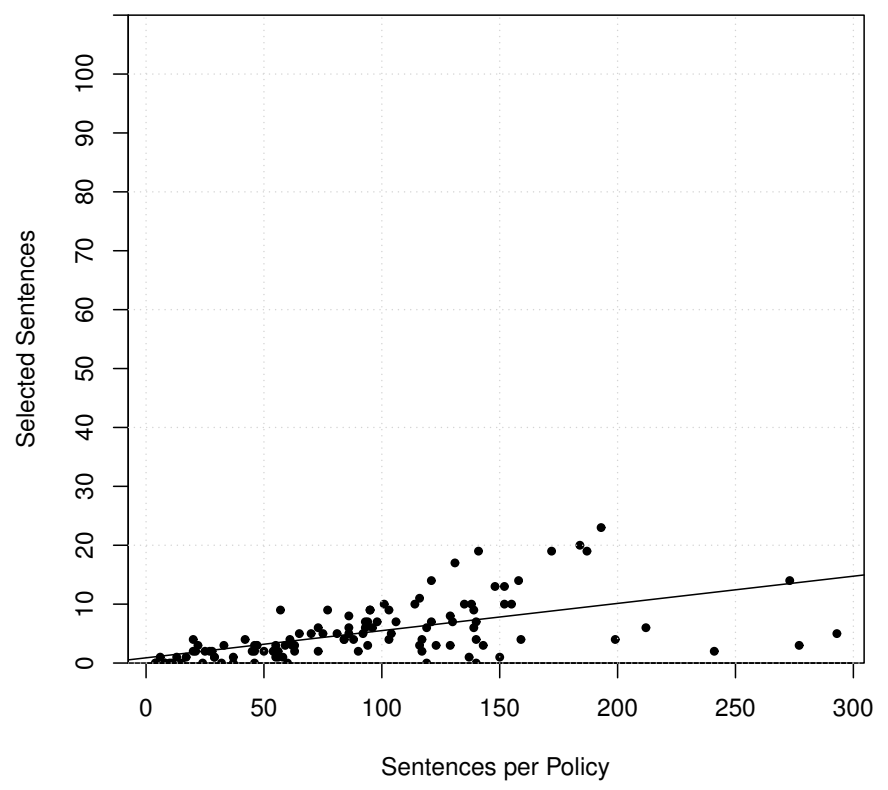

Figure 9. Results for experiment 2 for query 1 after considering contextual keywords. 
Table 11. Results for DOOP validation for query 2, experiment 4.

\begin{tabular}{ccccccccccc}
\hline \multirow{2}{*}{ 'Cookie' } & \multicolumn{3}{c}{$\mathbf{0 . 5}$} & \multicolumn{3}{c}{$\mathbf{0 . 7 5}$} & \multicolumn{3}{c}{$\mathbf{1}$} \\
\cline { 2 - 10 } & Total & Diff & \% & Total & Diff & \% & Total & Diff & \% \\
\hline No & 360 & 78 & $21.67 \%$ & 415 & 91 & $21.93 \%$ & 465 & 110 & $23.66 \%$ \\
Yes & 1689 & 550 & $32.56 \%$ & 1563 & 497 & $31.80 \%$ & 1689 & 550 & $32.56 \%$ \\
\hline
\end{tabular}

Table 12. Results for DOOP validation Query 1, Experiment 2 with contextual keywords.

\begin{tabular}{|c|c|c|c|c|c|c|c|c|c|}
\hline \multirow{2}{*}{$\begin{array}{l}\text { 'Contextual } \\
\text { Keywords' }\end{array}$} & \multicolumn{3}{|c|}{0.5} & \multicolumn{3}{|c|}{0.75} & \multicolumn{3}{|c|}{1} \\
\hline & TS & SS & Coverage & TS & SS & Coverage & TS & SS & Coverage \\
\hline No & 91.7 & 27.99 & $30.99 \%$ & 91.7 & 27.99 & $30.99 \%$ & 91.7 & 27.99 & $30.99 \%$ \\
\hline Yes & 91.7 & 5.12 & $5.57 \%$ & 91.7 & 5.12 & $5.57 \%$ & 91.7 & 5.12 & $5.57 \%$ \\
\hline
\end{tabular}

\section{Conclusions and Future Work}

Privacy policies play an important part in informing users about their privacy concerns. As the world becomes more interconnected and online, security threats prompt users to become more privacy aware, making online privacy policies the primary documents for users making informed decisions. These policies are long and difficult for most users to understand and are infrequently read, presenting a challenge for users. Previous attempts at creating machine readable policies have had limited success as they placed the onerous task of crafting these policies on businesses. This paper proposed a novel approach to reducing the amount of text a user has to read using a domain ontology and NLP to identify key areas of the policies that the user should read to address their concerns and take appropriate action. The approach consisted of constructing DOOP, a domain ontology for online privacy policies, validated against CMU's OPP-115 data set of annotated policies by domain experts. DOOP resulted in $69 \%, 99 \%$, and $96 \%$ reductions in reading for the 3 sample questions, and on average it would take about $45 \mathrm{~s}$ to read the relevant sentences (11 on average). By comparison, the average time to read privacy policies is estimated to be 29-32 min [10]. Furthermore, the vocabulary was mapped to the queries stored in the ontology. This allows ontology developers to propose additional insight into related queries and their associated vocabulary. The development of DOOP showed the usefulness of domain ontologies when applied to privacy policies, and also demonstrated a cost-effective way of maintaining and expanding it in the future.

In some of the recent studies performed by the authors, we found that the length of privacy policies that have been generated after the enforcement of regulations such as the General Data Protection Regulation has increased. These new regulations include the traditional recommended sections, such as the ones used in this paper, in addition to new sections such as contact information. Therefore, the proof-of-concept tool proposed in this study is applicable to the new and old versions of privacy policies. Furthermore, the competency questions we used in this study are relevant in the context of new regulations and best practices. The proposed tool can be improved by adding more competency questions to the ontology, using new and larger privacy policy corpus, and considering more recent privacy policies that were generated after the enforcement of regulations such as the General Data Protection Regulation.

Author Contributions: Conceptualization, D.A.A., R.D. and B.N.; methodology, D.A.A., R.D. and B.N.; software, D.A.A.; validation, D.A.A.; formal analysis, D.A.A., R.D. and B.N.; data curation, D.A.A.; writing-original draft preparation, D.A.A.; writing-review and editing, R.D. and B.N.; supervision, R.D. and B.N.; funding acquisition, R.D. All authors have read and agreed to the published version of the manuscript. 
Funding: This research was funded by the Natural Sciences and Engineering Research Council of Canada (NSERC). Nous remercions le Conseil de recherches en sciences naturelles et en gnie du Canada (CRSNG) de son soutien.

Institutional Review Board Statement: Not applicable.

Informed Consent Statement: Not applicable.

Data Availability Statement: Not applicable.

Conflicts of Interest: The authors declare no conflict of interest.

\section{References}

1. Madden, M.; Rainie, L. Americans' Attitudes about Privacy, Security and Surveillance. 2015. Available online: http://www. pewinternet.org/2015/05/20/americans-attitudes-about-privacy-security-and-surveillance/ (accessed on 9 November 2021).

2. Jensen, C.; Potts, C. Privacy Policies as Decision-Making Tools: An Evaluation of Online Privacy Notices. In Proceedings of the 2004 Conference on Human Factors in Computing Systems (CHI 2004), Vienna, Austria, 24-29 April 2004; Volume 6, pp. 471-478. [CrossRef]

3. Ten Steps to Develop a Multilayered Privacy Notice. 2006. Available online: https://www.huntonprivacyblog.com/wp-content/ uploads/sites /18/2012/07/Centre-10-Steps-to-Multilayered-Privacy-Notice.pdf (accessed on 9 November 2021).

4. Munur, M.; Branam, S.; Mrkobrad, M. Best PRACTICES in Drafting Plain-Language and Layered Privacy Policies. 2012. Available online: https:/ /iapp.org/news/a/2012-09-13-best-practices-in-drafting-plain-language-and-layered-privacy/ (accessed on 9 November 2021).

5. Westin, A.F. Privacy and Freedom; Atheneum: New York, NY, USA, 1967; p. 7.

6. Privacy Online: Fair Information Practices in the Electronic Marketplace. 2000. Available online: https://www.ftc.gov/reports/ privacy-online-fair-information-practices-electronic-marketplace-federal-trade-commission (accessed on 9 November 2021).

7. Regulation (EU) 2016/679 of the European Parliament and of the Council of 27 April 2016 on the Protection of Natural Persons with Regard to the Processing of Personal Data and on the Free Movement of Such Data, and Repealing Directive 95/46/EC (General Data Protection Regulation). 2016. Available online: http://eur-lex.europa.eu/legal-content/EN/TXT/PDF/?uri=CELEX: 32016R0679\&from=EN (accessed on 9 November 2021).

8. Personal Information Protection and Electronic Documents Act. 2000. Available online: http://laws-lois.justice.gc.ca/eng/acts/ P-8.6/index.html (accessed on 9 November 2021).

9. Digital Privacy Act. 2015. Available online: http://laws-lois.justice.gc.ca/eng/annualstatutes/2015_32/page-1.html (accessed on 9 November 2021).

10. Obar, J.A.; Oeldorf-Hirsch, A. The biggest lie on the internet: Ignoring the privacy policies and terms of service policies of social networking services. Inf. Commun. Soc. 2020, 23, 128-147. [CrossRef]

11. Mcdonald, A.M.; Reeder, R.W.; Kelley, P.G.; Cranor, L.F. A comparative study of online privacy policies and formats. In Proceedings of the 9th International Symposium on Privacy Enhancing Technologies (PETS '09), Seattle, WA, USA, 5-7 August 2009.

12. Milne, G.R.; Culnan, M.J. Strategies for reducing online privacy risks: Why consumers read (or don't read) online privacy notices. J. Interact. Mark. 2004, 18, 15-29. [CrossRef]

13. Wilson, S.; Schaub, F.; Dara, A.A.; Liu, F.; Cherivirala, S.; Leon, P.G.; Andersen, M.S.; Zimmeck, S.; Sathyendra, K.M.; Russell, N.C.; et al. The creation and analysis of a website privacy policy corpus. In Proceedings of the 54th Annual Meeting of the Association for Computational Linguistics (Volume 1: Long Papers), Berlin, Germany, 7-12 August 2016; Volume 1, pp. $1330-1340$.

14. Cranor, L.F.; Langheinrich, M.; Marchiori, M. A P3P preference exchange language 1.0 (APPEL1. 0). W3C Work. Draft $2002,15$. Available online: https:/ / ci.nii.ac.jp/naid/10027364004/ (accessed on 9 November 2021).

15. Cranor, L.F. P3P: Making privacy policies more useful. IEEE Secur. Priv. 2003, 1, 50-55. [CrossRef]

16. Lämmel, R.; Pek, E. Understanding privacy policies. Empir. Softw. Eng. 2013, 18, 310-374. [CrossRef]

17. Terms of Service; Didn't Read (ToS;DR). 2012. Available online: https://www.theverge.com/2012/8/14/3241572/tos-dr-termsof-service (accessed on 9 November 2021).

18. Zimmeck, S.; Bellovin, S.M. Privee: An architecture for automatically analyzing web privacy policies. In Proceedings of the 23rd USENIX Security Symposium (USENIX Security 14), San Diego, CA, USA, 20-22 August 2014; pp. 1-16.

19. Sadeh, N.; Acquisti, R.; Breaux, T.D.; Cranor, L.F.; Mcdonalda, A.M.; Reidenbergb, J.R.; Smith, N.A.; Liu, F.; Russellb, N.C.; Schaub, F.; et al. The Usable Privacy Policy Project: Combining Crowdsourcing, Machine Learning and Natural Language Processing to Semi-Automatically Answer Those Privacy Questions Users Care About; Carnegie Mellon University: Pittsburgh, PA, USA, 2013; pp. 1-24.

20. Ramanath, R.; Schaub, F.; Wilson, S.; Liu, F.; Sadeh, N.; Smith, N.A. Identifying relevant text fragments to help crowdsource privacy policy annotations. In Proceedings of the Second AAAI Conference on Human Computation and Crowdsourcing, Pittsburgh, PA, USA, 2-4 NOvember 2014.

21. Wilson, S.; Schaub, F.; Ramanath, R.; Sadeh, N.; Liu, F.; Smith, N.A.; Liu, F. Crowdsourcing Annotations for Websites' Privacy Policies: Can It Really Work? In Proceedings of the 25th International Conference on World Wide Web. International World Wide Web Conferences Steering Committee, Montreal, QC, Canada, 11-15 May 2016; pp. 133-143. 
22. Harkous, H.; Fawaz, K.; Lebret, R.; Schaub, F.; Shin, K.G.; Aberer, K. Polisis: Automated analysis and presentation of privacy policies using deep learning. In Proceedings of the 27th \{USENIX\} Security Symposium (\{USENIX\} Security 18, Baltimore, MD, USA, 15-17 August 2018; pp. 531-548.

23. Harkous, H.; Fawaz, K.; Shin, K.G.; Aberer, K. Pribots: Conversational privacy with chatbots. In Proceedings of the Twelfth Symposium on Usable Privacy and Security (\{SOUPS $\})$ 2016, Denver, CO, USA, 22-24 June 2016.

24. Zaeem, R.N.; German, R.L.; Barber, K.S. Privacycheck: Automatic summarization of privacy policies using data mining. ACM Trans. Internet Technol. (TOIT) 2018, 18, 53. [CrossRef]

25. Bui, D.; Shin, K.G.; Choi, J.M.; Shin, J. Automated Extraction and Presentation of Data Practices in Privacy Policies. Proc. Priv. Enhancing Technol. 2021, 2021, 88-110. [CrossRef]

26. Srinath, M.; Sundareswara, S.N.; Giles, C.L.; Wilson, S. PrivaSeer: A Privacy Policy Search Engine. In International Conference on Web Engineering; Springer: Berlin/Heidelberg, Germany, 2021; pp. 286-301.

27. Uschold, M.; Gruninger, M. Ontologies and semantics for seamless connectivity. ACM Sigmod Rec. 2004, 33, 58-64. [CrossRef]

28. Bermejo, J. A Simplified Guide to Create an Ontology; Madrid University: Madrid, Spain, 2007.

29. Malheiros, Y.; Freitas, F. A Method to Develop Description Logic Ontologies Iteratively Based on Competency Questions: An Implementation. ONTOBRAS 2013, 142-153. Available online: http:/ / citeseerx.ist.psu.edu/viewdoc/download?doi=10.1.1.403.2 $087 \&$ rep $=$ rep $1 \&$ type $=$ pdf\#page $=142$ (accessed on 9 November 2021).

30. Noy, N.F.; McGuinness, D.L. Ontology Development 101: A Guide to Creating Your First Ontology; Technical Report KSL-01-05 and SMI-2001-0880, Stanford Knowledge Systems Laboratory and Stanford Medical Informatics; Stanford University: Stanford, CA, USA, 2001.

31. Suárez-Figueroa, M.C.; Gómez-Pérez, A.; Fernández-López, M. The NeOn Methodology for Ontology Engineering. In Ontology Engineering in a Networked World; Suárez-Figueroa, M.C., Gómez-Pérez, A., Motta, E., Gangemi, A., Eds.; Springer: Berlin/Heidelberg, Germany, 2012; pp. 9-34. [CrossRef]

32. Musen, M.A. The protégé project: A look back and a look forward. AI Matters 2015, 1, 4-12. [CrossRef] [PubMed]

33. Brank, J.; Grobelnik, M.; Mladenić, D. A survey of ontology evaluation techniques. In Proceedings of the Conference on Data Mining and Data Warehouses, Houston, TX, USA, 22-26 August 2005; pp. 166-170. [CrossRef]

34. Liu, K.; Hogan, W.R.; Crowley, R.S. Natural Language Processing methods and systems for biomedical ontology learning. J. Biomed. Inform. 2011, 44, 163-179. [CrossRef] [PubMed]

35. Velardi, P.; Fabriani, P.; Missikoff, M. Using text processing techniques to automatically enrich a domain ontology. In Proceedings of the International Conference on Formal Ontology in Information Systems-Volume 2001, Ogunquit, ME, USA, 17-19 October 2001; pp. 270-284. [CrossRef]

36. Naik, M.P. A Survey on Semantic Document Clustering. In Proceedings of the Electrical, Computer and Communication Technologies (ICECCT), Coimbatore, India, 5-7 March 2015; pp. 1-10.

37. Grüninger, M.; Fox, M.S. Methodology for the Design and Evaluation of Ontologies. In Proceedings of the Workshop on Basic Ontological Issues in Knowledge Sharing (IJCAI-95), Montreal, QC, USA, 19-20 August 1995. Available online: http:/ / citeseerx.ist.psu.edu/viewdoc/ citations?doi=10.1.1.44.8723 (accessed on 10 August 2021).

38. Fridman Noy, N.; Hafner, C.D. The State of the Art in Ontology Design. AI Mag. 1997, 18, 53-74. [CrossRef]

39. Stanford Center for Biomedical Informatics Research, Stanford University School of Medicine. 2017. Available online: https: // protege.stanford.edu/products.php (accessed on 9 November 2021).

40. Audich, D.A.; Dara, R.; Nonnecke, B. Extracting keyword and keyphrase from online privacy policies. In Proceedings of the 2016 Eleventh International Conference on Digital Information Management (ICDIM), Porto, Portugal, 19-21 September 2016; pp. 127-132. [CrossRef]

41. Guntamukkala, N.; Dara, R.; Grewal, G. A Machine-Learning Based Approach for Measuring the Completeness of Online Privacy Policies. In Proceedings of the 2015 IEEE 14th International Conference on Machine Learning and Applications (ICMLA), Miami, FL, USA, 9-11 December 2015; pp. 289-294. [CrossRef]

42. OECD Guidelines on the Protection of Privacy and Transborder Flows of Personal Data. 2015. Available online: https://www. oecd.org/internet/ieconomy/oecdguidelinesontheprotectionofprivacyandtransborderflowsofpersonaldata.htm\#part2 (accessed on 9 November 2021).

43. Tsarkov, D.; Horrocks, I. FaCT++ description logic reasoner: System description. Autom. Reason. 2006, 4130, $292-297$.

44. Glimm, B.; Horrocks, I.; Motik, B.; Stoilos, G.; Wang, Z. HermiT: An OWL 2 Reasoner. J. Autom. Reason. 2014, 53, 245-269. [CrossRef]

45. Sirin, E.; Parsia, B.; Grau, B.C.; Kalyanpur, A.; Katz, Y. Pellet: A practical OWL-DL reasoner. Web Semant. Sci. Serv. Agents World Wide Web 2007, 5, 51-53. [CrossRef] 